\section{'V-55 A', VARIEDAD DE MAÍZ DE GRANO AMARILLO PARA LOS VALLES ALTOS DE MÉXICO}

\section{'V-55 A', A MAIZE VARIETY OF YELLOW GRAIN FOR MEXICAN HIGLANDS}

\author{
Alejandro Espinosa Calderón ${ }^{1}$, Margarita \\ Tadeo Robledo ${ }^{2^{\star}}$, Noel Gómez Montiel ${ }^{3}$, Mauro \\ Sierra Macías ${ }^{4}$, Juan Virgen Vargas ${ }^{1}$, Artemio \\ Palafox Caballero ${ }^{4}$, Filiberto Caballero \\ Hernández ${ }^{5}$, Gricelda Vázquez Carrillo ${ }^{1}$, \\ Flavio A. Rodríguez Montalvo ${ }^{4}$, Roberto \\ Valdivia Bernal ${ }^{6}$, Israel Arteaga Escamilla ${ }^{2}$ \\ e Isaías González Rojo ${ }^{2}$
}

\begin{abstract}
${ }^{1}$ Campo Experimental Valle de México, Instituto Nacional de Investigaciones Forestales, Agrícolas y Pecuarias (INIFAP), Km 13.5 Carretera Los Reyes - Texcoco. 56250, Coatlinchan, Texcoco, Estado de México, México. ${ }^{2}$ Ingeniería Agrícola, Facultad de Estudios Superiores Cuautitlán, Universidad Nacional Autónoma de México (FESC-UNAM), Carretera Cuautitlán - Teoloyucán, Km 2.5. 54714, Cuautitlán Izcalli, Estado de México. ${ }^{3}$ Campo Experimental Iguala, INIFAP. ${ }^{4}$ Campo Experimental Cotaxtla, INIFAP. ${ }^{5}$ Campo Experimental Valle de Apatzingan, INIFAP. ${ }^{6}$ Universidad Autónoma Nayarit.
\end{abstract}

*Autor para correspondencia: tadeorobledo@yahoo.com .

En los Valles Altos de la Mesa Central de México ubicados en altitudes de 2200 a $2600 \mathrm{~m}$, se cultivan cerca de $1.5 \mathrm{mi}$ llones de hectáreas con maíz (Zea mays L.), superficie que representa $20 \%$ de la establecida en el país con esta especie (Turrent, 1994; Ávila et al., 2009). De esta extensión, 800 mil hectáreas se cultivan en condiciones de temporal (secano), el cual generalmente inicia tarde, lo que restringe la fecha de siembra y la productividad del cultivo.

En el Estado de México se siembran 600 mil hectáreas de maíz, y se estima que en 300 mil la productividad es baja $\left(1.2 \mathrm{t} \mathrm{ha}^{-1}\right)$, principalmente por insuficiente lluvia y por siembras tardías que frecuentemente hacen que el cultivo sea afectado por heladas tempranas porque muchas de las variedades usadas son tardías. Una opción para estas condiciones ambientales es usar variedades mejoradas de ciclo corto, y adaptadas a las condiciones agroclimáticas disponibles. Sin embargo, la oferta de variedades mejoradas de este tipo es escasa, de modo que por lo general se siembran variedades criollas; las anteriores variedades mejoradas para temporal en Valles Altos son: 'V-26 A' (Cuapiaxtla), liberada en 1981 por el Instituto Nacional de Investigaciones Agrícolas (INIA) y 'Amarillo Zanahoria', liberada en 1990 por el Instituto de Investigación y Capacitación Agropecuaria, Acuícola y Forestal del Estado de México (ICAMEX), ambas con uso restringido actualmente (Espinosa et al., 2008).

México requiere incrementar la producción de maíz de grano amarillo para subsanar la demanda y reducir la importación anual de más de 10 millones de toneladas de grano entero y quebrado (Espinosa et al., 2008), cantidad que se usa para: elaborar alimentos forrajeros, extraer almidones, en la industria cerealera y botanera, así como para otros usos industriales. Alrededor de 2.3 millones de toneladas son procesadas por la Industria de Derivados Químicos y Alimenticios del Maíz (Tadeo y Espinosa, 2004; Tadeo et al., 2010). Mantener o aumentar la importación de maíz amarillo procesado será muy difícil para México debido a que en los Estados Unidos, principal proveedor de este producto, hay una fuerte demanda de maíz amarillo para producir etanol. En los mercados a futuro se considera que el precio internacional de maíz se mantendrá elevado y probablemente se incrementará (Espinosa et al., 2008).

Ante esta situación, la variedad mejorada de polinización libre 'V-55 A' de grano amarillo representa una alternativa para cubrir la necesidad de variedades mejoradas en México, que además ofrece mayor seguridad de producción por su ciclo vegetativo precoz. Esta variedad fue liberada en 2009 por el Campo Experimental Valle de México, perteneciente al Instituto Nacional de Investigaciones Forestales, Agrícolas y Pecuarias (INIFAP), y fue inscrita en el Catálogo Nacional de Variedades Vegetales (CNVV) con el número de registro definitivo: MAZ - 1092 - 100910, otorgado en 2010 por el Servicio Nacional de Inspección y Certificación de Semillas (SNICS). Esta nueva variedad mejorada es de ciclo precoz (136 d a madurez fisiológica) y tolerante al acame; su grano es de textura cristalina y rinde de 5.0 a $7.5 \mathrm{t} \mathrm{ha}^{-1}$. Por su precocidad puede sembrarse desde finales de mayo hasta fechas retrasadas como finales de junio. Además de ser una opción para siembra en condiciones de temporal limitado, representa una oportunidad para abastecer la demanda pecuaria de maíz amarillo (Espinosa et al., 2008, 2010; Tadeo et al., 2010).

'V-55 A' se obtuvo por recombinación durante dos ciclos de un compuesto balanceado de 21 líneas S2, de semilla amarilla, generadas a partir de siete híbridos de cruza simple. Tres líneas de cada híbrido fueron inicialmente seleccionadas por su precocidad y por el color amarillo intenso y textura cristalina del grano. Las cruzas simples, 
y posteriormente las líneas derivadas de éstas, fueron seleccionadas por su rendimiento y precocidad en ensayos de rendimiento efectuados en la Facultad de Estudios Superiores Cuautitlán de la Universidad Nacional Autónoma de México (FESC-UNAM), en Cuautitlán, México, conjuntamente con materiales del Centro Internacional de Mejoramiento de Maíz y Trigo (CIMMYT) y otros híbridos desarrollados en la propia FESC-UNAM y por el INIFAP. Después de recombinar el compuesto balanceado por dos ciclos, se aplicaron dos ciclos de selección masal para ganar en precocidad a madurez fisiológica, tolerancia al acame y textura cristalina del grano.

La mazorca de 'V-55 A' tiene 14 hileras de grano de textura cristalina y color amarillo (Figura 1). El grano es pequeño (28 g/100 granos) y de textura dura (13\% de flotantes), apto para las industrias de la masa-tortilla y de botanas. Sus contenidos de aceite (4.4\%) y proteína (10.2\%), así como de lisina $(0.377 \%)$, triptófano $(0.042 \%)$ y carotenos totales (41 $\mathrm{mg} \mathrm{kg}^{-1}$ ), permiten producir tortillas de buena calidad nutricia (Espinosa et al., 2010). El coeficiente de rendimiento maíz/tortilla de 1.42, valor que es bueno según el Laboratorio de Calidad de Maíz del INIFAP. Por ello, esta variedad constituye una alternativa para obtener productos alimenticios con características sensoriales aceptables para el consumidor, así como por aportar carotenos que funcionan como antioxidantes.

Las plantas de 'V-55 A' no forman hijos (macollos) ni tallos estériles; su altura es de 197 a $210 \mathrm{~cm}$ y la mazorca de 79 a $88 \mathrm{~cm}$ de altura. La mazorca mide de 14.0 a $14.7 \mathrm{~cm}$ de largo; la forma de la espiga, definida por el ángulo formado entre el eje principal y las ramas laterales en el tercio inferior de la espiga, es de compacta a muy compacta; las ramas laterales del tercio inferior de la espiga son rectilíneas y muy largas (mas de $25 \mathrm{~cm}$ ). La hoja de la mazorca principal es de color verde normal a verde oscuro. El tallo es de diámetro medio, que a la altura de la mazorca principal mide de 15.1 a $20.0 \mathrm{~mm}$.

'V-55 A' puede ser producida en el ciclo agrícola primavera-verano en localidades del Valle de México como Cuautitlán Izcalli, Texcoco y Zumpango, así como en localidades de los Estados de Tlaxcala y Puebla, en altitudes de 2100 a 2650 $\mathrm{m}$. Su rendimiento promedio de grano es de $5605 \mathrm{~kg} \mathrm{ha}^{-1}$, rendimiento que supera al de la variedad 'Amarillo Zanahoria' que en los años 2007 al 2009 produjo $2679 \mathrm{~kg} \mathrm{ha}^{-1}$. En evaluaciones efectuadas en 2007 en las localidades Mazapa, Francisco Villa, Xalpatlahuaya y Huamantla, Tlaxcala, y en Calimaya, México, todas en altitudes mayores de $2400 \mathrm{~m}$, 'V-55 A' tuvo un rendimiento promedio de $4387 \mathrm{~kg} \mathrm{ha}^{-1}$.

El Campo Experimental Valle de México (CEVAMEX) multiplica y mantiene las semillas de esta variedad en las

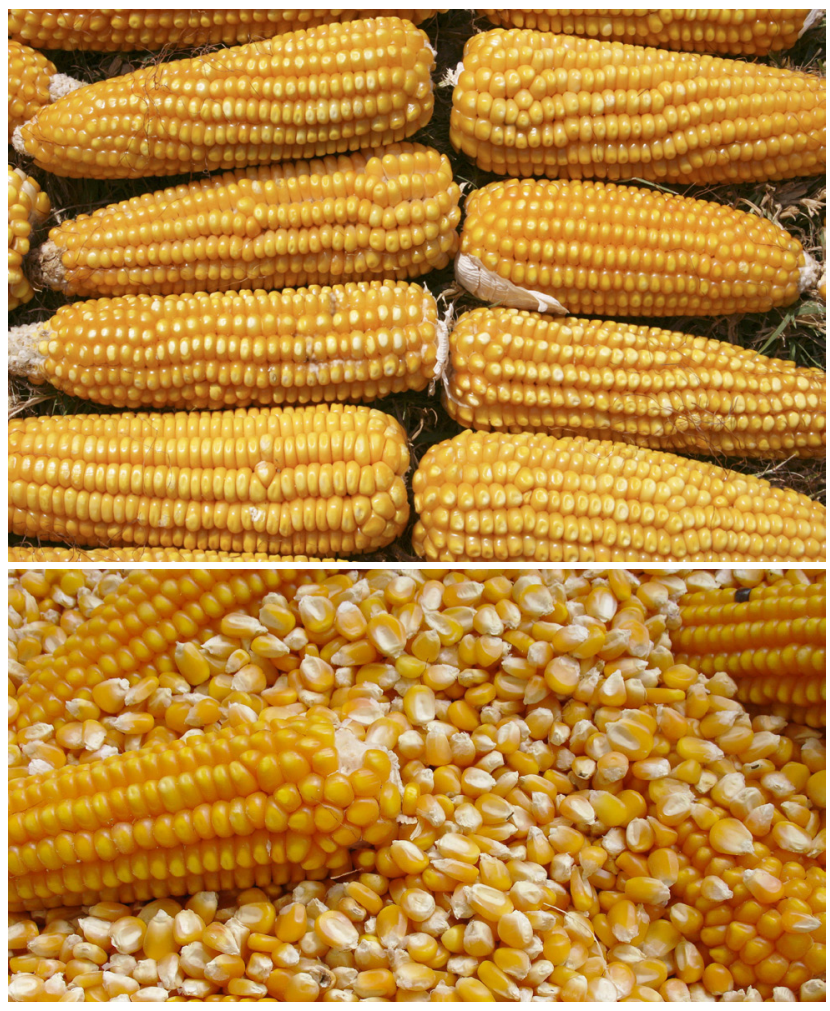

Figura 1. Mazorcas y grano de la variedad de maíz 'V-55 A'.

categorías original, básica y registrada. Esta última categoría de semilla puede ser adquirida por productores de semilla, como lo hicieron varias empresas semilleras para incrementarla durante 2010, para así abastecer las necesidades de siembra en los Valles Altos.

\section{BIBLIOGRAFÍA}

Ávila P M A, J L Arellano V, J Virgen V, A J Gámez V (2009) H-52 híbrido de maíz para Valles Altos de La Mesa Central de México. Agric. Téc. Méx. 35:237-240.

Espinosa C A, M Tadeo R, N Gómez M, M Sierra M, J Virgen V, A Palafox C, F Caballero H, I Arteaga E, E I Canales I, G Vázquez C, Y Salinas M (2010) V-55 A: variedad mejorada de grano amarillo y ciclo precoz para Valles Altos de México. In: Memoria Técnica No. 11, Día de Campo CEVAMEX 2010. Coatlinchán, México. pp:27-28.

Espinosa C A, M Tadeo R, A Turrent F, N Gómez M, M Sierra M, F Caballero H, R Valdivia B, F Rodríguez M (2008) El potencial de las variedades nativas y mejoradas de maíz. Ciencias 9293:118-125.

Turrent F A (1994) Plan de Investigación del Sistema Maíz-Tortilla en la Región Centro. CIRCE, INIFAP, SARH. Publicación Especial No. 12, Chapingo, México.

Tadeo R M, A Espinosa C (2004) Producción de semilla y difusión de variedades e híbridos de maíz de grano amarillo para Valles Altos de México. Rev. FESC Divulg. Científ. Multidisc. 14:5-10.

Tadeo R M, A Espinosa C, R Valdivia B, N Gómez M, M Sierra M, B Zamudio G (2010) Vigor de las semillas y productividad de variedades de maíz. Agron. Mesoam.21:31-38. 